\title{
Journal of Management and Business Education
}

\section{EFECTOS DE LA AUTOEVALUACIÓN Y DE LA EVALUACIÓN $360^{\circ}$ EN EL PROCESO DE ENSEÑANZA- APRENDIZAJE}

\section{EFFECTS OF SELF-ASSESSMENT AND 360 DEGREE FEEDBACK ON TEACHING-LEARNING PROCESS}

\author{
$\mathrm{M}^{\mathrm{a}}$ Isabel Barba-Aragón \\ Universidad de Murcia
}

2018

Vol.1 Num. 3

226-239

\section{RESUMEN}

Este trabajo destaca la importancia que tiene la evaluación como parte del proceso de enseñanza-aprendizaje y pretende mostrar su utilidad tanto para el profesor como para el estudiante. Para ello se han diseñado dos proyectos de innovación docente. El primero se centra en la autoevaluación y el segundo en la evaluación $360^{\circ}$. Los resultados del primer proyecto demuestran que la autoevaluación contribuye a la mejora de la calificación final del estudiante. En el segundo proyecto se comprueba que el alumnado está satisfecho con la evaluación $360^{\circ}$ y, además, muestra una satisfacción mayor con esta forma de valoración que con la evaluación tradicional realizada sólo por el docente. Con respecto a las desviaciones en las notas dadas por los distintos evaluadores, éstas se mueven entre el $0 \%$ y el $41 \%$. De estos proyectos se deduce que el uso de nuevas formas de evaluación del trabajo de los alumnos universitarios tiene un efecto positivo sobre su aprendizaje y sobre su satisfacción.

\section{PALABRAS CLAVE}

Innovación docente, autoevaluación, evaluación 360ํㅜ proceso de aprendizaje, enseñanza universitaria

\section{ABSTRACT}

This paper highlights the importance of evaluation as part of the teachinglearning process and aims to show its usefulness for both the teacher and the student. To this end, two teaching innovation projects have been designed. The first focuses on self-assessment and the second on the 360 degree feedback.

Barba-Aragon, M. I. (2018). Efectos de la autoevaluación y de la evaluación 360ํe en el proceso de enseñanza-aprendizaje. Journal of Management and Business Education, 1(3), 226-239. https://doi.org/10.35564/jmbe.2018.0016 
The results of the first project show that self-assessment contributes to the improvement of the student's final grade. In the second project, it is verified that the students are satisfied with the 360 degree feedback and, in addition, they show greater satisfaction with this form of assessment than with the traditional assessment carried out only by the teacher. With respect to the deviations in the scores given by the different evaluators, they move between $0 \%$ and $41 \%$. From these projects it can be deduced that the use of new ways of evaluating the work of university students has a positive effect on their learning and on their satisfaction.

\section{KEYWORDS}

Teaching innovation, self-assessment, 360 degree feedback, learning process, university education.

\section{INTRODUCIÓN}

En la actualidad la educación universitaria está sufriendo un proceso de cambio muy significativo. Esta transformación se debe a factores tales como: la convergencia europea, nuevos planes de estudios o nuevos créditos computables (ECTS) (Inda Caro, Álvarez González, \& Álvarez Rubio, 2008). Las directrices de Bolonia han favorecido un cambio en la cultura docente dentro de la enseñanza universitaria que ha llevado a la puesta en práctica de numerosas acciones de innovación docente en el contexto de la educación superior (Mauri, Coll, \& Onrubia, 2007). De hecho, innovar en docencia se ha convertido casi en una exigencia institucional para adaptarse a la era del conocimiento y a los estándares del Espacio Europeo de Educación Superior (Castilla-Polo, 2011). Este proceso se encuentra sometido a muchas presiones y no pocas contradicciones, por lo que hay que ser consciente de las limitaciones reales que existen en el ámbito de la innovación y mejora de la docencia universitaria (Zabalza, 2004). No obstante, parece lógico suponer que cualquier cambio o innovación debe ir dirigido a una mejora y, por tanto, la innovación docente debe buscar una mejora de la calidad de la enseñanza y la satisfacción de profesores y alumnos con dicho proceso de educación.

También hay que tener presente que, tal y como señalan Sánchez-Vidal, Cegarra-Leiva y Rodríguez-Arnaldo (2017), los profesores universitarios muestran una formación limitada en técnicas pedagógicas (a diferencia de otros colectivos docentes) y que, la mayoría, no tiene experiencia educativa cuando son contratados. Ello da lugar a que suelan seguir la metodología de enseñanza de sus predecesores, de modo que el docente universitario usa principalmente la clase magistral como forma de transmitir conocimientos a los estudiantes.

Teniendo en cuenta que el profesorado debe ayudar a sus alumnos a aprender de forma proactiva, involucrándose en su propio proceso de aprendizaje, se están desarrollando dos proyectos de innovación docente para tratar de mejorar los resultados de aprendizaje y la satisfacción de los alumnos que cursan las asignaturas de Gestión de RRHH I y Gestión de RRHH II del Grado en Relaciones Laborales y Recursos Humanos de la Facultad de Ciencias del Trabajo de la Universidad de Murcia. 
El primer proyecto, denominado "La autoevaluación como mecanismo docente de mejora del aprendizaje en la asignatura de Gestión de RRHH I", pretende aplicar la metodología de la autoevaluación por parte del alumnado a partir del uso de las Tecnologías de la Información y la Comunicación (TIC), en concreto, por medio de las herramientas disponibles a través del Aula Virtual de la Universidad de Murcia. En la educación superior es necesario un entorno educativo innovador con herramientas que permitan, tanto al alumnado como al profesorado, emplear nuevas metodologías en el proceso de enseñanzaaprendizaje a través de las TIC (Martín, León, \& García, 2014). La utilización de las TIC puede ayudar a promover una mayor calidad en los resultados de aprendizaje y satisfacción de los estudiantes (Sánchez-Vidal, Cegarra-Leiva, \& Rodríguez Arnaldo, 2017).

El segundo proyecto, titulado "Evaluación $360^{\circ}$ en el estudio de casos de la asignatura Gestión de Recursos Humanos II", tiene como principal objetivo el diseño y puesta en marcha de la metodología de la evaluación 360ํㅡ en la asignatura de Gestión de Recursos Humanos II buscando un aumento de la satisfacción de sus alumnos.

Ambos proyectos comparten una temática común: la evaluación. Ello se debe, por un lado, a que la calidad de la educación depende de muchos factores, tales como la calidad del profesorado, la estructura del plan de estudios, la gestión administrativa e institucional o las infraestructuras y el equipamiento, pero uno de los factores que destaca especialmente es la evaluación que se aplica durante todo el proceso de enseñanza-aprendizaje. Y, por otro, a que la evaluación es de gran utilidad tanto para el alumno como para el profesor. Al alumno le permite saber cómo y en qué medida ha asimilado los conocimientos propuestos o hasta qué nivel han desarrollado sus habilidades gracias al aprendizaje. Al docente le proporciona información sobre la eficacia de las estrategias pedagógicas propuestas en las clases lo que le ayuda a decidir si debe continuar con la misma didáctico o debe cambiar e innovar para que sus alumnos alcancen un mayor aprendizaje (Camacho-Gironda, 2014).

Asimismo, ambos proyectos apuestan por centrarse en la persona que aprende ya que la investigación demuestra la necesidad de involucrar a los estudiantes en su propio aprendizaje a través tanto de la autoeavaluación como de la evaluación de sus compañeros (Ndoye, 2017). Tras el profundo cambio que se está produciendo en el contexto universitario parece claro que lo importante ahora es ayudar a adquirir conocimientos, ayudar a aprender. El profesor se convierte así en un guía y el alumno pasa de desempeñar un papel meramente pasivo (simple receptor del conocimiento) a ser sujeto activo del proceso (Inda Caro, Álvarez González, \& Álvarez Rubio, 2008).

A continuación, para ambos proyectos se exponen los objetivos previstos, la metodología empleada y los resultados alcanzados. 


\section{PROYECTO 1: LA AUTOEVALUACIÓN COMO MECANISMO DOCENTE DE MEJORA DEL APRENDIZAJE EN LA ASIGNATURA DE GESTIÓN DE RRHH I.}

\section{Justificación y objetivos}

La evaluación es una tarea compleja y tiene una gran importancia en el proceso de enseñanza-aprendizaje. La evaluación es una parte fundamental, del proceso mismo de la educación, y, es indisoluble con el aprendizaje y la asimilación de conocimientos (Camacho-Gironda, 2014). Según Contreras (2004), evaluar es un proceso que implica obtener buena información respecto a los dominios de los alumnos (conocimientos, aptitudes, habilidades, comportamientos, etc.), establecer juicios de valor y tomar decisiones. Según Inda Caro, Álvarez González y Álvarez Rubio (2008), la evaluación ha de ser una parte integrante del aprendizaje y nunca un añadido. La evaluación tiene que resultar motivadora y productiva tanto para los estudiantes como para el docente.

Normalmente, la evaluación va dirigida del profesor al estudiante. Sin embargo, este proceso puede ser más eficiente cuando es el alumno el que se "autoevalúa", utilizando para ello una serie de estrategias didácticas, guías de preguntas, batería de pruebas y otras técnicas pedagógicas, que son elaboradas con el apoyo del docente y se aplican en el aula (Camacho-Gironda, 2014). Además, el Espacio Europeo de Educación Superior ha introducido cambios metodológicos en la docencia universitaria que promueve actividades académicas orientadas al autoaprendizaje, entre las que se encuentra la autoevaluación (Martín, León, \& García, 2014).

La autoevaluación es un proceso donde los estudiantes valoran su rendimiento a través de criterios estandarizados y predeterminados y se involucran en una autorregulación dinámica y en una autoreflexión (Bourke \& Mentis, 2011).

La autoevaluación se puede emplear en diferentes momentos temporales. Puede utilizarse en evaluaciones iniciales o pruebas de nivel al comienzo de una asignatura, lo que permiten al estudiante conocer sus carencias antes de iniciar el estudio de una materia, en evaluación continua, lo que ayuda a que el alumnado conozca su rendimiento a lo largo del curso y/o en evaluación final, que posibilita que el estudiante sea consciente del grado en que ha alcanzado los objetivos propuestos una vez finalizado el periodo académico (García-Beltrán et al., 2016).

Esta técnica exige que existan unos criterios de evaluación muy claros y objetivos (que pueden ser negociados conjuntamente entre profesor-alumno) pero también requiere de un alto grado de confianza del profesor hacia el alumno. La autoevaluación puede contribuir a desarrollar la capacidad crítica del alumno y favorecer su independencia y creatividad y resulta una estrategia clave para educar en la responsabilidad y para aprender a valorar, criticar y a reflexionar sobre el proceso de enseñanza y aprendizaje individual (Calatayud, 2002).

La literatura ha analizado el papel que la autoevaluación puede desempeñar en el aprendizaje del estudiante. Dochy, Segers y Sluijsmans (1999) revisaron 
63 estudios y encontraron que la autoevaluación promueve la autorreflexión, favorece la resolución de problemas y mejora la responsabilidad frente al propio aprendizaje. Por su parte, Bourke y Mentis (2011) analizaron los beneficios de la autoevaluación y hallaron que aumentaba las oportunidades de aprendizaje. Sharma et al. (2016) analizaron la mejora en el rendimiento académico de un grupo de estudiantes después de seguir un proceso de autoevaluación y obsevaron que los alumnos mejoraron sus calificaciones.

Teniendo en cuenta que este proyecto de innovación docente va dirigido a alumnos que cursan la asignatura de Gestión de RRHH I, se pretende que estos estudiantes desarrollen su autonomía, responsabilidad, flexibilidad y que utilicen las TICs. Competencias clave para futuro profesional en el ámbito de la empresa y poder insertarse con éxito en el mercado laboral actual.

\section{Metodología}

La nueva metodología se utilizó en el grupo 2 de Gestión de RRHH I durante los años académicos 2016/2017 y 2017/18. Esta asignatura se imparte en el primer cuatrimestre del segundo curso del Grado en Relaciones Laborales y Recursos Humanos de la Facultad de Ciencias del Trabajo de la Universidad de Murcia. Las principales características del curso se observan en la tabla 1.

Tabla 1. Principales características de la asignatura y el curso

\begin{tabular}{|l|l|}
\hline Título de la asignatura & Gestión de Recursos Humanos I \\
\hline Grado & Relaciones Laborales y Recursos Humanos \\
\hline Créditos europeos & 6 \\
\hline Curso del grado & 2 \\
\hline Número de docentes & 1 \\
\hline Número de alumnos & $84(16 / 17)$ \\
& $73(17 / 18)$ \\
\hline
\end{tabular}

La metodología del proyecto se estructuró en torno a una serie de actividades que se describen a continuación:

- La profesora explicó a los estudiantes en qué consistía la metodología de autoevaluación y cómo y cuándo se iba a aplicar. En concreto, se llevó a cabo a través del Aula Virtual de la Universidad de Murcia. Al igual que la mayoría de entonos virtuales de aprendizaje/enseñanza más utilizados en la actualidad, como WebCT, Moodle o Claroline (García-Beltrán et al., 2016), el Aula Virtual posee una herramienta (denominada en este caso "exanet") que permite el planteamiento de pruebas de respuesta objetiva.

- La profesora impartió los conocimientos relativos a cada uno de los temas de la asignatura. Concluida la explicación de cada tema, se puso a disposición de los estudiantes un examen tipo test compuesto 10 preguntas con cuatro posibles respuestas y una única opción correcta. Las preguntas en blanco no penalizaban mientras que cada pregunta mal contestada restaba $1 / 3$ de la puntuación de una respuesta bien contestada. 
- Se habilitó un periodo de 2 días en el que los estudiantes podían, de forma voluntaria, realizar su autoevaluación contestando, en un periodo muy limitado de tiempo, el conjunto de preguntas del tema, obteniendo inmediatamente su calificación.

- En la clase posterior a la fecha de realización de cada autoevaluación, la profesora dedicó unos minutos a la resolución de las preguntas propuestas y a la aclaración de dudas relacionadas con su contenido.

- Al finalizar la impartición de la asignatura se analizó en qué medida los resultados de autoevaluación de los estudiantes estaban correlacionados con la calificación final obtenida en la asignatura.

\section{Resultados}

El grupo 2 de segundo curso del Grado en Relaciones Laborales y Recursos humanos tenía 84 alumnos matriculados en la asignatura de Gestión de RRHH I en el curso 2016/17. De estos 84 alumnos, 10 no se presentaron al examen final de la asignatura. En la tabla 2 se muestran los resultados obtenidos:

Tabla 2. Resultados de la autoevaluación en Gestión de RRHH I (curso 2016/17)

\begin{tabular}{|c|c|c|c|c|}
\hline & \multicolumn{2}{|l|}{$\mathbf{N}(\%)$} & $\begin{array}{l}\text { Calificación } \\
\text { final }\end{array}$ & \\
\hline $\begin{array}{l}\text { Correlación entre nota } \\
\text { media de autoevaluación } \\
\text { y calificación final }\end{array}$ & \multicolumn{2}{|l|}{$74(100)$} & 4,43 & $\begin{array}{l}\text { Correlación } \\
\text { bivariada } \\
0,64^{\star *}\end{array}$ \\
\hline \multirow{2}{*}{$\begin{array}{l}\text { Diferencia de calificación } \\
\text { final entre alumnos que } \\
\text { han hecho } \\
\text { alguna/ninguna prueba } \\
\text { autoevaluación }\end{array}$} & $\begin{array}{l}\text { Alguna } \\
\text { autoevaluación }\end{array}$ & $\begin{array}{l}57 \\
(77,03)\end{array}$ & 4,67 & \multirow[b]{2}{*}{$\begin{array}{l}t \text { de Student } \\
2,91^{* *}\end{array}$} \\
\hline & $\begin{array}{l}\text { Ninguna } \\
\text { autoevaluación }\end{array}$ & $\begin{array}{l}17 \\
(22,97)\end{array}$ & 3,59 & \\
\hline \multirow{2}{*}{$\begin{array}{l}\text { Diferencia de calificación } \\
\text { final entre alumnos que } \\
\text { han hecho } \\
\text { mayoría/minoría pruebas } \\
\text { autoevaluación }\end{array}$} & $\begin{array}{l}\text { Mayoría } \\
\text { autoevaluación }\end{array}$ & $\begin{array}{l}46 \\
(62,16)\end{array}$ & 4,94 & \multirow[b]{2}{*}{$\begin{array}{l}t \text { de Student } \\
3,93^{* *}\end{array}$} \\
\hline & $\begin{array}{l}\text { Minoría } \\
\text { autoevaluación }\end{array}$ & $\begin{array}{l}28 \\
(37,84)\end{array}$ & 3,59 & \\
\hline \multirow{2}{*}{$\begin{array}{l}\text { Diferencia de calificación } \\
\text { final entre alumnos que } \\
\text { han hecho } \\
\text { aprobado/suspendido } \\
\text { pruebas autoevaluación }\end{array}$} & $\begin{array}{l}\text { Aprobado } \\
\text { autoevaluación }\end{array}$ & $\begin{array}{l}22 \\
(29,73)\end{array}$ & 5,84 & \multirow{2}{*}{$\begin{array}{l}\text { t de Student } \\
5,47^{\star *}\end{array}$} \\
\hline & $\begin{array}{l}\text { Suspenso } \\
\text { autoevaluación }\end{array}$ & $\begin{array}{c}52 \\
(70,27)\end{array}$ & 3,83 & \\
\hline
\end{tabular}

En el curso 2017/18 el grupo 2 de segundo curso del Grado en Relaciones Laborales y Recursos humanos tenía 73 alumnos matriculados en la asignatura de Gestión de RRHH I. El estudio se realizó sobre 62 alumnos al no presentarse 11 al examen final de la asignatura. En la tabla 3 se muestran los resultados obtenidos: 
Tabla 3. Resultados de la autoevaluación en Gestión de RRHH I (curso 2017/18)

\begin{tabular}{|c|c|c|c|c|}
\hline & \multicolumn{2}{|l|}{ N (\%) } & $\begin{array}{l}\text { Calificación } \\
\text { final }\end{array}$ & \\
\hline $\begin{array}{l}\text { Correlación entre nota } \\
\text { media de } \\
\text { autoevaluación y } \\
\text { calificación final }\end{array}$ & \multicolumn{2}{|l|}{$62(100)$} & 5,62 & $\begin{array}{l}\text { Correlación } \\
\text { bivariada } \\
0,65^{\star *}\end{array}$ \\
\hline \multirow{2}{*}{$\begin{array}{l}\text { Diferencia de } \\
\text { calificación final entre } \\
\text { alumnos que han } \\
\text { hecho alguna/ninguna } \\
\text { prueba autoevaluación }\end{array}$} & $\begin{array}{l}\text { Alguna } \\
\text { autoevaluación }\end{array}$ & $\begin{array}{l}59 \\
(95,16)\end{array}$ & 5,74 & \multirow[b]{2}{*}{$\begin{array}{l}t \text { de Student } \\
2,18^{*}\end{array}$} \\
\hline & $\begin{array}{l}\text { Ninguna } \\
\text { autoevaluación }\end{array}$ & $3(4,84)$ & 3,39 & \\
\hline \multirow{2}{*}{$\begin{array}{l}\text { Diferencia de } \\
\text { calificación final entre } \\
\text { alumnos que han } \\
\text { hecho mayoría/minoría } \\
\text { pruebas } \\
\text { autoevaluación }\end{array}$} & $\begin{array}{l}\text { Mayoría } \\
\text { autoevaluación }\end{array}$ & $\begin{array}{l}54 \\
(87,10)\end{array}$ & 5,93 & \multirow[b]{2}{*}{$\begin{array}{l}\text { t de Studen } \\
3,64^{* *}\end{array}$} \\
\hline & $\begin{array}{l}\text { Minoría } \\
\text { autoevaluación }\end{array}$ & $\begin{array}{l}8 \\
(12,90)\end{array}$ & 3,58 & \\
\hline \multirow{2}{*}{$\begin{array}{l}\text { Diferencia de } \\
\text { calificación final entre } \\
\text { alumnos que han } \\
\text { hecho } \\
\text { aprobado/suspendido } \\
\text { pruebas } \\
\text { autoevaluación }\end{array}$} & $\begin{array}{l}\text { Aprobado } \\
\text { autoevaluación }\end{array}$ & $\begin{array}{l}35 \\
(56,45)\end{array}$ & 6,37 & \multirow[b]{2}{*}{$\begin{array}{l}\text { t de Student } \\
4,00^{\star \star}\end{array}$} \\
\hline & $\begin{array}{l}\text { Suspenso } \\
\text { autoevaluación }\end{array}$ & $\begin{array}{l}27 \\
(43,55)\end{array}$ & 4,66 & \\
\hline
\end{tabular}

Los resultados de las pruebas estadísticas ponen claramente de manifiesto que la realización de pruebas de autoevaluación contribuye a la mejora de las calificaciones finales de los alumnos del grupo 2 de la asignatura "Gestión de Recursos Humanos I" tanto en el curso 2016/17 como en el 2017/18. En primer lugar, se observa que existe un alto grado de asociación entre la nota media de las pruebas de autoevaluación y la calificación final que obtiene el alumno en la asignatura, como demuestra la alta correlación entre ambas (0,64 y 0,65 para cada curso, respectivamente). Y, en segundo lugar, cuando se analizan las diferencias por grupos, se observa que el alumno obtiene una calificación final significativamente superior:

- cuando realiza una o más pruebas de autoevaluación que cuando no hace ninguna (4,67 vs. 3,59 en el curso $2016 / 17 ; 5,74$ vs. 3,39 en el curso 2017/18),

- cuando realiza la mayoría de las pruebas de autoevaluación que cuando se presenta sólo a una minoría (4,94 vs. 3,59 en el curso 2016/17; 5,93 vs. 3,58 en el curso $2017 / 18$ ),

- cuando aprueba las pruebas de autoevaluación que cuando las suspende (5,84 vs. 3,83 en el curso $2016 / 17 ; 6,37$ vs. 4,66 en el curso 2017/18). 


\section{PROYECTO 2: EVALUACIÓN 360 EN EL ESTUDIO DE CASOS DE LA ASIGNATURA GESTIÓN DE RECURSOS HUMANOS II}

\section{Justificación y objetivos}

La evaluación 360 grados (también conocida como evaluación integral) es una herramienta que se utiliza en el ámbito de la gestión del talento para valorar de forma integral el desempeño de un individuo/trabajador. Se trata de una técnica que se utiliza frecuentemente en las empresas para evaluar el rendimiento y las competencias de sus empleados (Gómez-Mejía, Balkin, \& Cardy, 2016). De hecho es una técnica que se explica en el primer tema de la asignatura "Gestión de Recursos Humanos II" y consiste en que las actuaciones de un individuo sean valoradas por todos los agentes de su ámbito (en la empresa el desempeño del trabajador es evaluado por su superior, sus compañeros, sus subordinados, clientes o él mismo). Según Dolan et al. (2007), esta diversidad de información puede aportar un visión más realista pero plantea el inconveniente de tener que conciliar la dispersión de los criterios. La evaluación $360^{\circ}$ es especialmente válida como sistema que aporta retroalimentación al evaluado y parece conectar con la filosofía de la gestión de la calidad total y generar un mayor nivel de satisfacción de los evaluados. Según Pérez (2015), los beneficios que se pueden obtener al aplicar este sistema de evaluación son, entre otros: obtener información desde diferentes perspectivas de cada individuo, conseguir información más fiable, reducir los prejuicios y sesgos que pueden producirse cuando la valoración es responsabilidad de una sola persona, mejorar la percepción de equidad, transparencia y justicia en los procesos de evaluación. Proporciona retroalimentación constructiva y totalmente anónima

Al proponer su aplicación en el ámbito docente en lugar de en el empresarial, el estudiante pasa a ocupar el papel que el trabajador desempeña en el contexto empresarial y los evaluadores pasan a ser el profesor, los compañeros y el propio alumno.

El interés de este proyecto es doble porque, por un lado, el alumnado va a poder aplicar en el aula una técnica que se emplea habitualmente en el área de gestión de recursos humanos a nivel empresarial (y que de hecho es objeto de estudio en la asignatura), lo que le va a servir de campo experimental para conocer la problemática de su uso y va a facilitar su incorporación al mercado laboral. Por otro lado, el alumnado va a actuar como sujeto receptor de la evaluación 360․ Con ello va a poder experimentar los dos puntos de vista y va a conocer de primera mano las ventajas e inconvenientes del método.

Asimismo, el proyecto va a permitir que los estudios de casos resueltos por el alumnado sean valorados por los diferentes grupos de interés implicados en la asignatura (profesores, compañeros, autoevaluación), obteniéndose así una evaluación más objetiva lo que, en esencia, es el propósito fundamental de esta técnica de valoración del rendimiento.

Con este proyecto se trata de que:

- el estudiante conozca la valoración que de su trabajo (plasmado en la resolución de los estudios de casos que se proponen en la asignatura) hacen sus compañeros, profesores y él mismo. 
- El estudiante identifique sus fortalezas y debilidades como estudiante frente a las competencias que la asignatura ha definido como fundamentales para su superación.

- Se consiga una evaluación más objetiva del trabajo del estudiante al estar basada en las percepciones de diferentes evaluadores.

- El estudiante sea no sólo un agente evaluado en la asignatura sino que conozca y asuma el papel de evaluador.

La literatura ha estudiado cómo la autoevaluación y la evaluación a compañeros incide en el proceso de enseñanza-aprendizaje. Según estos estudios, ambos tipos de evaluación refuerzan una atmósfera de aprendizaje autorregulado que influye positivamente en la adquisición de conocimientos (Ndoye, 2017). Logan (2009) encontró que estas técnicas de evaluación mejoraban el aprendizaje del estudiante ayudándolo a desarrollar un pensamiento crítico y a tomar conciencia de su papel como receptor de conocimiento. De Grez, Valcke, \& Roozen (2012) señalaron que los estudiantes conseguían un mayor aprendizaje si se sentían comprometidos en la evaluación de sus compañeros. Por su parte, Planas Lladó et al. (2013) demostraron que la evaluación a compañeros mejoraba la motivación, aumentaba la confianza en las habilidades personales y tenía un efecto positivo en el compromiso de los estudiantes. Mientras que Ndoye (2017) indicó que la autoevaluación y la evaluación de compañeros permite a los estudiantes aplicar sus habilidades evaluadoras y los ayuda a estar mejor preparados para comprometerse con el aprendizaje a lo largo de toda su vida.

Dado el importante papel que, según la literatura, juegan tanto la autoevaluación como la evaluación de compañeros en el proceso de enseñanzaaprendizaje, es esencial saber qué opinan los estudiantes de estas formas de valoración de su trabajo (Ndoye, 2017). Sin embargo, poco se ha estudiado de las percepciones que el alumnado tiene sobre la evaluación $360^{\circ}$. Por ello, este proyecto analiza el grado de satisfacción que el alumnado muestra ante la evaluación $360^{\circ}$.

\section{Metodología}

Esta metodología se va a utilizar en todos los grupos de Gestión de RRHH II durante el año académico 2017/2018. Esta asignatura se imparte en el segundo cuatrimestre del segundo curso del Grado en Relaciones Laborales y Recursos Humanos de la Facultad de Ciencias del Trabajo de la Universidad de Murcia. Las principales características del curso se observan en la tabla 4. 
Tabla 4. Principales características de la asignatura y el curso

\begin{tabular}{|l|l|}
\hline Título de la asignatura & Gestión de Recursos Humanos I \\
\hline Grado & Relaciones Laborales y Recursos Humanos \\
\hline Créditos europeos & 6 \\
\hline Curso del grado & 2 \\
\hline Número de docentes & 5 \\
\hline Número de alumnos: & \\
Grupo 1 & 87 \\
Grupo 2 & 76 \\
Grupo 3 & 74 \\
Total & 237 \\
\hline
\end{tabular}

La metodología del proyecto se estructura en torno a una serie de actividades que se describen a continuación:

- El profesorado explica en qué consiste la evaluación 360 (éste es uno de los contenidos del tema 1 de la asignatura de Gestión de RRHH II).

- El profesorado ofrece una serie de directrices a los estudiantes sobre cómo se va a aplicar la evaluación 360 grados al contexto del aula y a la valoración concreta de los estudios de casos que se ven en la parte práctica de la asignatura.

- El caso objeto de estudio es resuelto de forma individual por cada estudiante con los conocimientos adquiridos tras la explicación del tema que ha realizado el profesor. El profesor indicará a los alumnos que deben estudiar previamente los contenidos del tema pero que en el momento de la resolución del caso no podrán consultar los materiales de estudio.

- El caso resuelto se da a un compañero para que lo valore (ponga una puntuación) en función de los conocimientos que ha adquirido tras las clases teórica pero sin poder consultar tampoco los materiales existentes sobre el tema.

- El estudiante recibe su caso puntuado por un compañero y lo revisa y puntúa comparándolo en esta ocasión con los contenidos de los materiales que se han facilitado para estudiar el tema.

- Tras estas dos evaluaciones (compañeros y el propio estudiante) es el profesor el que valorará/puntuará el caso resuelto por el alumno.

- La nota final del caso objeto de estudio será la media de las tres evaluaciones recibidas.

- Al final del curso, se analiza

1) el grado de satisfacción del estudiante con la metodología $360^{\circ}$. Para ello se ha diseñado un cuestionario en el que solicita al alumno que indique su grado de acuerdo ( $1=$ totalmente en desacuerdo: $5=$ totalmente de acuerdo) con una serie de ítemes relativos a la satisfacción generada por la aplicación del sistema de evaluación $360^{\circ}$.

2) la desviación existente entre las 3 puntuaciones que ha recibido la resolución de cada caso objeto de estudio. 


\section{Resultados}

En los tres grupos de segundo curso del Grado en Relaciones Laborales y Recursos humanos hay en total 245 alumnos matriculados en la asignatura de Gestión de RRHH II en el curso 2017/18. De estos 245 alumnos, 162 (69 grupo A, 46 grupo B y 47 grupo C) han participado en la evaluación $360^{\circ}$ y han contestado el cuestionario de satisfacción.

Los resultados de le encuesta demuestran que los estudiantes están satisfechos con la aplicación de un sistema de valoración de los casos prácticos de la asignatura basado en la técnica $360^{\circ}$. Ante la afirmación "En general, estoy satisfecho con la aplicación de este sistema de evaluación" se obtiene un valor medio de 4.02 sobre 5 . Ante la afirmación "Me siento más satisfecho con el sistema de evaluación $360^{\circ}$ " en comparación con una evaluación tradicional, los estudiantes puntúan con una media de 3.90 sobre 5 .

Analizando las desviaciones entre las tres calificaciones que se otorgan a cada caso práctico (la del compañero, autoevaluación del estudiante y la del profesor) se observa que la desviación mínima es de 0 puntos, es decir, hubo casos en las que las tres calificaciones coincidieron exactamente, y la desviación máxima es de 4.1 puntos ( $41 \%$ de la nota).

En general, los resultados arrojan que la nota más alta de las tres evaluaciones que recibe cada caso práctico corresponde a la otorgada mediante autoevaluación y que la nota más baja suele ser la que puntúa el docente.

\section{DISCUSIÓN, CONCLUSIONES E IMPLICACIONES}

La educación actual requiere de la transformación y aplicación de nuevos métodos y herramientas de enseñanza-aprendizaje (Estelles-Miguel et al., 2018). Con la llegada del Espacio Europeo de Educación Superior ha crecido la importancia de la evaluación y su necesidad de integración dentro de los mecanismos de aprendizaje en el contexto de la docencia universitaria (Inda Caro, Álvarez González, \& Álvarez Rubio, 2008). El uso de nuevas formas de evaluación parece ser un camino a seguir para mejorar los resultados de adquisición de conocimientos y habilidades y para aumentar la satisfacción. Sin embargo, se han realizado pocos estudios para analizar cómo estos nuevos métodos de evaluación afectan al aprendizaje y a la satisfacción de los alumnos. El proyecto 1 se enmarca dentro del uso de la metodología de la autoevaluación. Con esta metodología se espera que los estudiantes mejoren sustancialmente el nivel de asimilación de los nuevos conocimientos en el aula, mejorando así las competencias teóricas y procedimentales y propiciando actitudes positivas (Camacho-Gironda, 2014). Tras la aplicación del proyecto docente 1 en la asignatura de Gestión de RRHH I, se puede decir que existe una correlación positiva y significativa entre la nota de las autoevaluaciones y la calificación final del alumno. También se observan diferencias significativas en la nota final en Gestión de RRHH I, obteniendo notas superiores aquellos alumnos que realizaron más pruebas de autoevaluación respecto a los que hicieron menos. Por último, los resultados señalan que aquellos alumnos que superan las pruebas de autoevaluación a lo largo del cuatrimestre obtienen una calificación final más alta en la asignatura. Estos resultados coinciden con los alcanzados por otros estudios que observaron mejoras en el rendimiento académico de los 
estudiantes tras pasar por procesos de autoevaluación (Bourke \& Mentis, 2011; Sharma et al., 2016; Sánchez-Marín et al., 2018).

El proyecto 2 se encuadra dentro de la aplicación de un sistema de evaluación $360^{\circ}$. Con esta técnica se espera que el alumnado se encuentre más satisfecho con su evaluación de los casos prácticos de la asignatura de Gestión de RRHH Il al considerar que existe más objetividad y transparencia que cuando es puntuado por un solo evaluador. Los resultados de este proyecto confirman nuestras suposiciones. Los estudiantes muestran una mayor satisfacción con la técnica de la evaluación $360^{\circ}$ por sí misma y también comparándola con el sistema de evaluación tradicional en el que el peso de la valoración corresponde al docente. Al analizar las desviaciones entre las tres calificaciones otorgadas, se observa que en algunos casos las diferencias son importantes por lo que se debe buscar una solución para conseguir menos variación en las puntuaciones. De esta forma, la valoración de cada evaluador resultará más fiable y más acorde a la realidad. Los resultados de este proyecto corroboran conclusiones obtenidas por investigaciones previas, tales como las de Logan (2009), De Grez, Valcke, y Roozen (2012), Planas Lladó et al. (2013) o Ndoye (2017) que informan de mejoras en el proceso de enseñanza-aprendizaje cuando se emplea la autoevaluación y la evaluación de compañero como complemento de la valoración del docente.

\section{DECLARACIÓN DE CONFLICTO DE INTERESES/DECLARATION OF CONFLICTING INTERESTS}

Los autores declaran que no existen conflictos de intereses potenciales con respecto a la investigación, autoría y o publicación de este artículo.

\section{FINANCIACIÓN/FUNDING}

The authors received no financial support for the research, authorship, and/or publication of this article.

\section{ORCID iDs}

María Isabel Barba Aragón https://orcid.org/0000-0001-9387-5313

\section{REFERENCIAS}

Alfalla-Luque, R.; Medina-López, C. \& Arenas-Márquez, F. (2011). Mejorando la formación en Dirección de Operaciones: la visión del estudiante y su respuesta a diferentes metodologías docentes. Cuadernos de Economía y Dirección de la Empresa. 14. 40-52.

Bourke, R. \& Mentis, M. (2011). Self-assessment as a process for inclusion. International Journal of Inclusive Education. 17(8). 854-867.

Calatayud, A. (2002). La cultura autoevaluativa, piedra filosofal de la calidad en educación. Educadores. 204. 357-375.

Camacho Gironda, C.A. (2014). La autoevaluación en el aula Un método innovador para mejorar la calidad del proceso enseñanza-aprendizaje en la formación de maestras y maestros. Integra Educativa. VII(2). 159-172.

Castilla-Pozo, F. (2011). Calidad docente en el ámbito universitario: un estudio comparativo de las universidades andaluzas. Revista de Educación en Contabilidad, Finanzas y Administración de Empresas. 2. 157-172. 
Contreras, E. (2004). Evaluación de los aprendizajes universitarios. En Docencia universitaria. Orientaciones para la formación del profesorado. Documentos ICE (pp.129-152). Oviedo: Universidad de Oviedo.

De Grez, L., Valcke, M. \& Roozen, I. (2012). How effective are self- and peer assessment of oral presentation skills compared with teachers' assessments?. Active Learning in Higher Education. 13(2). 129-142.

Dochy, F., Segers, M. \& Sluijsmans, D. (1999). The use of self-, peer and coassessment in higher education: A review. Studies in Higher Education. 24(3). 331-350.

Dolan, S.L., Valle Cabrera, R., Jackson, S.E. \& Schuler, R.S. (2007). La gestión de los recursos humanos. Madrid: McGraw Hill.

Estelles-Miguel, S.; Peris-Ortiz, M.; Albarracín Guillem, JM.; \& Palmer Gato, ME. (2018). Nuevas formas de enseñanza y aprendizaje en dirección de producción y operaciones. Journal of Management and Business Education, 1(1). 69-79.

García-Beltrán, A.; Martínez, R.; Jaén, J.A. \& Tapia, S. (2016). La autoevaluación como actividad docente en entornos virtuales de aprendizaje/enseñanza. Revista de Educación a Distancia. 50(14)-.1-11.

Gómez-Mejía, L.R., Balkin, D.B. \& Cardy, R.L. (2016). Gestión de recursos humanos. Madrid: Pearson Educación.

Inda Caro, M., Álvarez González, S. \& Álvarez Rubio, R. (2008). Métodos de evaluación en la enseñanza superior. Revista de Investigación Educativa. 26(2). 539-552.

Logan, E. (2009). Self and peer assessment in action. Practioner Research in Higher Education. 3(1). 29-35.

Martín, A., León, C. \& García, A. (2014). Innovación docente para la integración de autoformación y autoevaluación en la plataforma webct. Revista de Medios y Educación. 44. 201-214.

Mauri, T., Coll, C. \& Onrubia, J. (2007). La evaluación de la calidad de los procesos de innovación docente universitaria. Una perspectiva constructivista. Red U. Revista de Docencia Universitaria, 1. Obtenido de http://www.redu.um.es/Red_U/1/

Ndoye, A. (2017). Peer / Self Assessment and Student Learning, International Journal of Teaching and Learning in Higher Education. 29(2). 255-269.

Pérez, O. (2015). ¿Qué es la evaluación de 360 grados y qué beneficios aporta a tu empresa?. Obtenido de http://blog.peoplenext.com.mx/que-es-laevaluacion-de-360-grados-y-que-beneficios-aporta-a-tu-empresa

Planas Lladó, A., Soley, L.F., Fraguell Sansbelló, R.M., Pujolras, G.A., Planella, J.P., Roura-Pascual, N. \& Moreno, L.M. (2013). Student perceptions of peer assessment: An interdisciplinary study. Assessment \& Evaluation in Higher Education. 39(5). 592-610.

Reinholz, D. (2016). The assessment cycle: A model for learning through peer assessment. Assessment \& Evaluation in Higher Education. 41(2). 301-315.

Sánchez-Marín, G.; Lucas-Pérez, M.E.; Carrasco-Hernández, A.J.; LozanoReina, G.; \& NicolásMartínez, C. (2018). The influence of self-assessment activities on student learning outcomes. Journal of Management and Business Education, 1(1). 28-38.

Sánchez-Vidal, M.E., Cegarra-Leiva, D. \& Rodríguez Arnaldo, O. (2017). Una experiencia de innovación pedagógica basada en la clase inversa y las nuevas 
tecnologías. Análisis de resultados de aprendizaje y satisfacción en un curso de la universidad. CEF. 8. 11-38.

Sharma, R.; Jain, A.; Gupta, N.; Garg, S.; Batta, M. \& Dhir, S.K. (2016). Impact of self-assessment by students on their learning. International Journal of Applied Basic Medical Research. 6(3). 226-229.

\section{Cita recomendada}

Barba-Aragon, M. I. (2018). Efectos de la autoevaluación y de la evaluación 360은 en el proceso de enseñanza-aprendizaje. Journal of Management and Business Education, 1(3), 226-239. https://doi.org/10.35564/jmbe.2018.0016 